\title{
Warfarin induced skin necrosis: a diagnostic challenge
}

\begin{abstract}
Warfarin is a commonly used oral anticoagulant in the treatment of many major chronic illnesses. Early diagnosis and discontinuation of warfarin are crucial for the better prognosis. We are sharing an interesting case of warfarin-induced skin necrosis to increase physician awareness as warfarin induced skin necrosis is difficult to differentiate from some other dermatologic conditions. This case also illustrates that a high degree of suspicion combined with clinical history, laboratory findings and sometimes skin biopsy are required to make diagnosis.
\end{abstract}

Volume 6 Issue I - 2017

\author{
Muhammad Tariq Shakoor,' Samia Ayub, ${ }^{2}$ \\ Zunaira Ayub ${ }^{3}$ \\ 'Hospitalist, Baptist Health Medical Center, USA \\ 2Pulmonary Critical Care Fellow, University of Arkansas for \\ Medical Science, USA \\ ${ }^{3}$ Medical Officer, Sir Ganga Ram Hospital, Pakistan
}

Correspondence: Muhammad Tariq Shakoor, Hospitalist, Baptist Health Medical Center, Little Rock, AR 72205, USA, Email mts2703@hotmail.com

Received: January 15, 2016 | Published: January 20, 2017

\section{Introduction}

Warfarin induced skin necrosis is a rare dermatologic complication of warfarin therapy. This dermatologic condition occurs in 0.01 to 0.1 percent of warfarin treated patients. Early recognition of the condition is crucial for prompt intervention but a high degree of suspicion is required to make diagnosis as the skin lesion can be challenging to differentiate from some other medical conditions. Well Informed clinicians can intervene quickly to alter the course and prevent its significant morbidity and potential mortality. ${ }^{1}$

\section{Case report}

A generally healthy 74-year-old male with past medical history of hypertension and recently diagnosed with paroxysmal atrial fibrillation, presented with left arm skin lesion for one week. According to the patient, the lesion started as petechia that progressed to ecchymosis and hemorrhagic bullae over last one week. Patient denied any other complaints. Home medications included metoprolol, hydrochlorothiazide and coumadin. Patient denied any history of trauma, similar lesions in the past or same lesion at any other place, recent sea food intake, diarrhea or chronic liver disease. His initial vitals were stable and physical examination showed left hemorrhagic, bullous and necrotic skin lesion spread in the whole left arm, more prominent in forearm area (Figure 1). His basic labs including $\mathrm{CBC}$, CMP were normal except INR of 2.8.

His warfarin was held and the patient was admitted for further work up. Our initial differential diagnosis included hematoma, pyoderma gangrenosum, venous gangrene, purple toe syndrome, cryofibrinogenemia, steven Johnson Syndrome, Vibrio Vulnificus Infection, hemangioma, pyoderma gangrenosum, heparin induced skin necrosis and Warfarin Induced Skin Necrosis. His protein C level was low on further work up. Bedside skin biopsy was performed which showed diffuse dermal microthrombi with endothelial cell damage and red cell extravasation with full-thickness coagulative necrosis (Figure 2), confirming diagnosis of warfarin induced skin necrosis. Patient was discharged home without warfarin and his skin lesion completely resolved with local wound care.

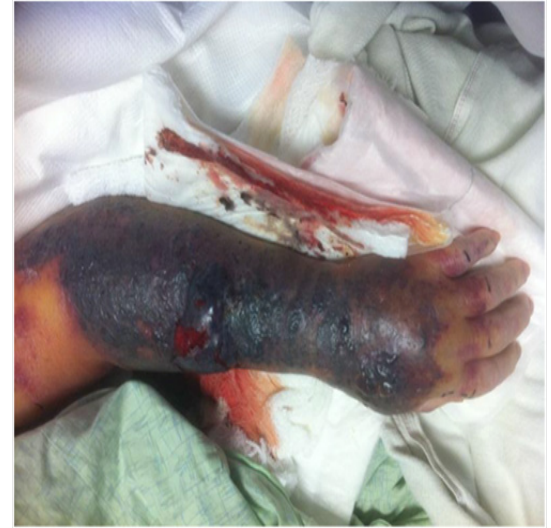

Figure I Left arm hemorrhagic, bullous and necrotic skin lesion spread in the whole arm, more prominent in forearm and dorsum of hand area.

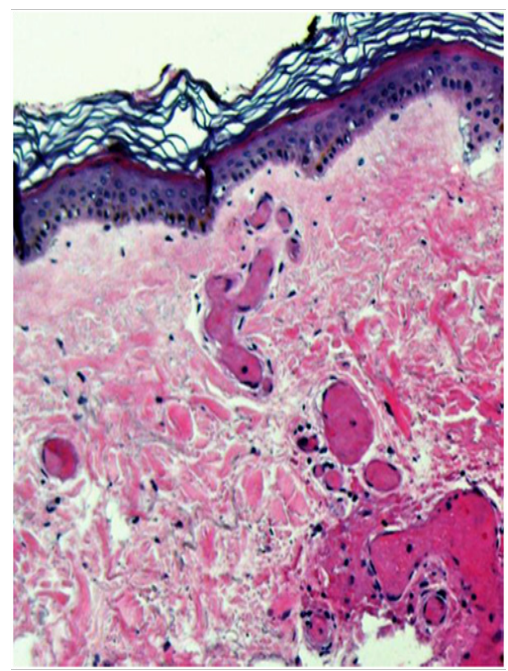

Figure 2 Skin biopsy showing diffuse dermal micro thrombi with endothelial cell damage and red cell extravasation with full-thickness coagulative necrosis. 


\section{Discussion}

Warfarin is a widely used anticoagulant. It works by inactivating vitamin K-dependent clotting factors II, VII, IX and X. At the same time, Vitamin $\mathrm{K}$ dependent proteins $\mathrm{C}$ and $\mathrm{S}$ are inactivated these are natural anticoagulants. 1 Protein $\mathrm{C}$ has half-life of 6hours as compared to Factor X and II, which have half-lives of 2-5days. Therefore, proteins $\mathrm{C}$ and $\mathrm{S}$ are depleted first, resulting in a relatively hypercoagulable state during the initial 24 to 48 hours of warfarin therapy. In some circumstances this leads to excessive clotting which causes dermal microthrombi resulting in skin necrosis. ${ }^{2}$ The risk factors include high loading doses of warfarin, prior deficiencies of proteins $\mathrm{C}$ and/or S and antithrombin III, and mutations in the methylenetetrahydrofolate reductase gene. ${ }^{3}$

The diagnosis of warfarin induced skin necrosis is made clinically. Skin biopsy can aid in diagnosis. It usually reveals clotting within blood vessels in the skin without any inflammation. Blood test for levels of protein $\mathrm{C}$ and protein $\mathrm{S}$ is important to determine any predisposing factors. ${ }^{3}$

The mainstay of treatment is to stop warfarin. If anticoagulation is required, heparin can be used. Sometimes Vitamin K is used to hasten the reversal of warfarin effects. If there is life-threatening coagulation then protein $\mathrm{C}$ concentrates can be used. ${ }^{2}$ Continued wound care and sometimes skin grafting is required to prevent further complications. ${ }^{4}$

\section{Conclusion}

We conclude that the lesions of warfarin-induced skin necrosis may be difficult to differentiate from similar skin conditions, but skin biopsy in conjunction with careful consideration of the clinical history, including time of onset, cutaneous distribution of the lesions, and laboratory findings, are essential for prompt diagnosis and patient treatment.

\section{Acknowledgements}

None.

\section{Conflict of interest}

The author declares no conflict of interest.

\section{References}

1. Nazarian R, Van Cott E, Zembowicz A, et al. Warfarin-induced skin necrosis. J Am Acad Dermatol. 2009;61(2):325-332.

2. Stephan M. Warfarin induced skin necrosis. British Journal of Hematology. 2004;126(5):628.

3. Byrne JS, Abdul Razak AR, Patchett S, et al. Warfarin skin necrosis associated with protein $\mathrm{S}$ deficiency and a mutation in the methylenetetrahydrofolate reductase gene. Clin Exp Dermatol. 2004;29(1):35-36.

4. Pourdeyhimi N, Bullard Z. Warfarin-induced skin necrosis. Hosp Pharm. 2014;49(11):1044-1048. 